\title{
A minimally invasive approach to spleen histopathology in dogs: A new method for follow-up studies of spleen changes in the course of Leishmania infantum infection
}

\author{
Silvana Ornelas Santos ${ }^{\mathrm{a}, \mathrm{b}}$, Jonathan L.M. Fontes ${ }^{\mathrm{a}}$, Daniela F. Laranjeira ${ }^{\mathrm{b}, \mathrm{c}}$, José Vassallo ${ }^{\mathrm{d}}$, \\ Stella Maria Barrouin-Melo ${ }^{\mathrm{b}, \mathrm{c}, * *}$, Washington L.C. dos-Santos ${ }^{\mathrm{a}, *}$ \\ a Fundação Oswaldo Cruz, Centro de Pesquisas Gonçalo Moniz, Salvador, BA, Brazil;, Brazil \\ ${ }^{\mathrm{b}}$ Laboratório de Infectologia Veterinária, Hospital de Medicina Veterinária, Universidade Federal da Bahia (UFBA), Salvador, BA, Brazil \\ ' Departmento de Anatomia, Patologia and Clínicas, Escola de Medicina Veterinária e Zootecnia, UFBA, Salvador, BA, Brazil \\ d Faculdade de Ciências Médicas, UNICAMP, Campinas, SP, Brazil
}

\section{A R T I C L E I N F O}

\section{Article history:}

Received 14 April 2016

Received in revised form 11 August 2016

Accepted 17 August 2016

\section{Keywords:}

Leishmania infantum

Dog

Canine visceral leishmaniasis

Spleen

Cell block

Fine-needle biopsy

Minimally invasive histopathology

\begin{abstract}
A B S T R A C T
Severe forms of zoonotic visceral leishmaniosis (ZVL) are associated with disruption of the spleen structure. However, the study of spleen histology requires splenectomy or necropsy. In this work, we present a minimally invasive cell-block technique for studying spleen tissue histology in dogs with ZVL. We examined 13 dogs with and seven dogs without Leishmania infantum infection. The dogs with Leishmania infection had a lower frequency of lymphoid follicles $(2 / 13$, Fisher's test, $P<0.02)$ and a higher density of plasma cells (score 3, Fisher's test, $P<0.02)$ than uninfected dogs $(5 / 7$ exhibiting lymphoid follicles and a plasma cell score of 1 ). The dogs with Leishmania infection also presented with granulomas (8/13) and infected macrophages (5/13). These differences in the histological presentations of spleen tissue from infected and uninfected dogs corresponded to changes observed in conventional histology. Hence, the cell-block technique described here may be used in the follow-up care and study of dogs with ZVL and other diseases in both clinical practice and research.
\end{abstract}

(C) 2016 Elsevier Ltd. All rights reserved.

\section{Introduction}

The spleen is the largest secondary lymphoid organ in mammals and is responsible for surveillance against blood-borne antigens [1]. This organ is the site of the final differentiation and homing of many leukocyte populations, including monocyte populations and memory B cells responsive to both T-dependent and T-independent type 1 and type 2 antigens [2,3]. The spleen also plays an important role in the course of neoplastic and infectious diseases [4,5]. For instance, absence of the spleen is associated with an increased risk of disseminated infections, and spleen infiltration is an important parameter for lymphoma staging in both humans and dogs $[5,6]$.

The spleen plays a central role in the course of zoonotic visceral leishmaniosis (ZVL) in humans and in dogs and is involved

\footnotetext{
* Corresponding author.

** Corresponding author at: Laboratório de Infectologia Veterinária, Hospital de Medicina Veterinária, Universidade Federal da Bahia (UFBA), Salvador, BA, Brazil.

E-mail addresses: barrouin@ufba.br (S.M. Barrouin-Melo),

wluis@bahia.fiocruz.br, wlcs25@gmail.com (W.L.C. dos-Santos).
}

throughout the course of the disease [7]. Spleen enlargement is an important clinical sign of chronic infection, so a spleen regression index is used as a marker of the therapeutic response in human ZVL [8]. Furthermore, the spleen may contribute to the progression of ZVL by (1) providing a permissive environment, thereby maintaining the parasite burden; (2) promoting anemia and thrombocytopenia through hypersplenism; and (3) modulating the immune response to favor parasite survival [9-11]. Recently, we and other groups have shown that disruption of splenic lymphoid tissue is associated with severe forms of ZVL in dogs [12-15]. Similar changes in spleen structure have also been reported in humans who died of the disease [16].

Unfortunately, most of the morphological changes in the spleen that are potentially relevant to the course of infectious or neoplastic disease can be studied only in specimens obtained by necropsy or splenectomy. A few years ago, we developed a technique for fine-needle aspiration spleen biopsy that proved to be safe for sensitive and specific diagnosis of Leishmania infection and allowed for the study of spleen cytology in dogs [17-19]. In that study, we observed that small and sparse fragments of spleen tissue remained in the midst of single-cell suspensions that were prepared by 
cytocentrifugation [19]. In the present work, we show that these spleen tissue fragments obtained by fine-needle biopsy can be used as a source of information about spleen morphology in dogs with ZVL. This information can be acquired using a cell-block technique for processing the fine-needle spleen aspirates.

In addition to the information obtained via conventional cytology, this cell-block technique allows spleen compartments to be studied and pathological structures, such as granulomas and blood vessel hyalinosis, to be identified. Because fine-needle aspiration biopsy is a well-tolerated and safe procedure in dogs under sedation [17], its combination with this cell-block technique may allow for sequential studies on the development, reversibility, and impact of spleen changes during the course of $L$. infantum infection and ZVL.

\section{Materials and methods}

\subsection{Ethical considerations}

All procedures were conducted in accordance with the Oswaldo Cruz Foundation guidelines for research in animals (http:// sistemas.cpqam.fiocruz.br/ceua/hiceuaw000.aspx) and with the manual for the surveillance and control of visceral leishmaniosis. All the tests were performed in the animals after authorization by the Control of Visceral Services of Camacari (Bahia State, Brazil, an area endemic for ZVL) (1 animal) or by their owners (all the other infected and control animals). This study was approved by the ethics committee for the use of animals in research (CPqGMFIOCRUZ, Ceua, license N.040/2005).

\subsection{Animals}

This was a convenience sample of 20 dogs of different breeds, genders, and estimated ages that were collected either by the Surveillance and Control of Visceral Services of Camacari (Bahia State, Brazil) or from the Veterinary School Hospital (Federal University of Bahia). Eight dogs from the hospital and five from Camacari had clinical signs of ZVL or positive laboratory tests for Leishmania infection. One animal from the hospital was healthy, having been admitted for castration, and was enrolled in the control group. Six other healthy animals were from a non-governmental animal shelter located in Salvador (Bahia State, Brazil, an area nonendemic for ZVL). All the animals were subjected to a clinical exam, serological testing for anti-Leishmania antibodies and culture of spleen aspirates for Leishmania isolation. These tests are further detailed below.

\subsection{Clinical data}

All animals were subjected to a clinical exam using a standardized form and were considered symptomatic if they had any of the following clinical signs, which are considered to be indicative of canine visceral leishmaniosis: alopecia, dermatitis, skin erosion, ulcerations, anemia (pale, light pink mucous membranes), conjunctivitis, onychogryphosis, weight loss, and lymph nodes larger than expected for the size of the animal.

\subsection{Anti-Leishmania antibody activity in the serum}

The presence of anti-Leishmania antibodies in the serum of all studied dogs was investigated using a classical indirect ELISA using soluble $L$. infantum antigens, as previously described [17]. Briefly, 96-well plates were coated with the antigen overnight at $4{ }^{\circ} \mathrm{C}$ (incubation with $100 \mathrm{~mL}$ per well of $5 \mathrm{mg}$ of protein per $\mathrm{mL}$ of $0.1 \mathrm{M}$ carbonate-bicarbonate solution, $\mathrm{pH} 9.6$ ). Non-specific antibody binding was blocked with $5 \%$ skimmed milk $(\mathrm{w} / \mathrm{v})$ in
$0.15 \mathrm{M}$ phosphate-buffered saline (PBS) solution, $\mathrm{pH} 7.4$, containing $0.05 \%$ Tween 20 (PBS-T) for $1 \mathrm{~h}$, at $37^{\circ} \mathrm{C}$. Control and test serum samples were added at a 1:400 dilution in $1 \%$ skimmed milk in PBS-T, with $100 \mathrm{~mL} /$ well, and incubated for $1 \mathrm{~h}$ at $37^{\circ} \mathrm{C}$. A peroxidase-conjugated rabbit anti-dog IgG (Sigma-Aldrich Chemical Co., St. Louis, Missouri, EUA) was added at a dilution of 1:5000 in $1 \%$ skimmed milk in PBS-T, with $100 \mathrm{~mL} /$ well, and incubated for $1 \mathrm{~h}$ at $37^{\circ} \mathrm{C}$. The enzymatic reaction was developed with ophenylenediamine (Sigma Chemical Co.) and stopped after $15 \mathrm{~min}$ with $25 \mathrm{~mL} /$ well of $4 \mathrm{~N} \mathrm{H}_{2} \mathrm{SO}_{4}$. Absorbance values were read at $490 \mathrm{~nm}$. The cut-off value for IgG antibodies was determined based on the receiver operating characteristic (ROC) curve using corrected absorbance values obtained for sera from $30 \mathrm{~L}$. infantum-infected dogs positive in serological and parasitological tests and from 71 healthy dogs living in non-endemic areas. All determinations were carried out in duplicate or triplicate, and mean values above the cut-off for IgG were considered to be positive results.

\subsection{Fine-needle spleen biopsy}

Fine-needle aspiration spleen biopsies were performed according to the technique previously described by Barrouin-Melo et al. (2006a). Briefly, dogs were sedated through intravenous injection of $0.5 \mathrm{mg} / \mathrm{kg}$ body weight acepromazine in the right lateral decubitus. Asepsis of the area that would be punctured was ensured using $2 \%$ iodized alcohol. A splenic puncture was made in the left flank of each animal near the caudal border of the last rib using a $40 \times 12-\mathrm{mm}$ needle coupled to a $20-\mathrm{mL}$ syringe [17]. Part of each splenic aspirate was cultivated in a biphasic culture medium for parasitological diagnosis, and part was dispensed into a $15-\mathrm{mL}$ nonadhering (propylene) tube containing $10 \%$ formalin and was used for the cell-block technique, as described below.

\subsection{Culture for parasitological diagnosis of Leishmania}

Samples obtained by spleen aspiration (approximately $100-200 \mu \mathrm{L}$ ) were cultured in a biphasic culture medium containing $1.5 \mathrm{~mL}$ of solid medium (blood agar) and $2 \mathrm{~mL}$ of Schneider's medium supplemented with $20 \%$ fetal bovine serum. The samples were kept in culture at $23^{\circ} \mathrm{C}$ and examined weekly by optical microscopy for four weeks or until they became positive [20].

\subsection{Cell-block preparation and analysis}

Spleen aspirates (approximately 100-200 $\mu \mathrm{L}$ ) were placed in non-adherent polypropylene tubes containing $14 \mathrm{~mL}$ of $10 \%$ formalin and fixed under agitation for $12-24 \mathrm{~h}$ at room temperature. The preparations were then centrifuged for $10 \mathrm{~min}$ at $1000 \mathrm{~g}$. The supernatant was removed, and the sediment was carefully placed in a small parcel made of filter paper wetted with formalin. The specimens were then dehydrated with progressive concentrations of ethanol and embedded in paraffin. Sections of 3- $\mu \mathrm{m}$ thickness were obtained for conventional microscopy and immunohistochemistry. For conventional histology, cell-block sections were stained with hematoxylin-eosin (H\&E), periodic acid-Schiff (PAS), and periodic acid-silver methenamine (PAS-M). Serial sections of the cell-block preparations were analyzed without knowledge of the parasitological or serological test results according to the following criteria:

\subsection{Splenic tissue features}

The number of spleen fragments; the sizes of smaller and larger spleen fragments; spleen compartments (red pulp, white pulp, periarteriolar lymphoid sheaths, lymphoid follicles, and marginal zone); trabeculae; blood vessels, including sheathed capillaries; and pathological structures (granulomas and Leishmania-infected 
Table 1

General characteristics of the dogs used in the study of spleen samples collected by fine-needle aspiration and the cell-block technique.

\begin{tabular}{|c|c|c|c|}
\hline \multirow[t]{2}{*}{ Evidence of Leishmania infection } & \multirow[t]{2}{*}{ Symptomatic ${ }^{a}$} & \multicolumn{2}{|c|}{ Evidence of Leishmania infection } \\
\hline & & NO & YES \\
\hline Positive serology, culture, and histology & YES & 0 & 3 \\
\hline Positive serology and culture & YES & 0 & 2 \\
\hline Positive serology and histology & $N A^{b}$ & 0 & 2 \\
\hline Positive serology & YES & 0 & 4 \\
\hline Positive serology & NA & 0 & 2 \\
\hline Negative serology, culture, and histology & NO & 7 & 0 \\
\hline
\end{tabular}

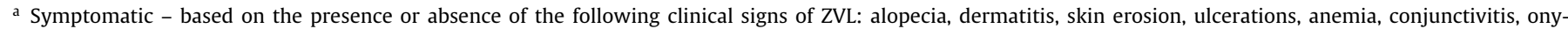
chogryphosis, weight loss, and lymphadenopathy.

b NA - information not available.

macrophages) were recorded as present or absent. Cells were characterized morphologically according to previously described criteria [12]: Lymphocytes - cells with round nuclei containing condensed chromatin, inconspicuous nucleoli, and scant cytoplasm. Plasma cells - cells with eccentric nuclei, heterochromatin dispersed around the edge in a pattern similar to that of the numerals on an analog clock face, and basophilic cytoplasm with clear perinuclear vacuoles. Macrophages - large cells with oval or reniform nuclei containing loosely packed chromatin surrounded by a rim of eosinophilic cytoplasm with indistinct edges. Granulocytes - cells with highly lobulated nuclei containing densely packed chromatin. Megakaryocytes - very large cells with wide and clearly defined cytoplasm and large, lobulated nuclei containing condensed chromatin. The densities of different cell populations present in the red pulp were scored as follows: $0=$ no cells, $1=$ isolated cells or small aggregates as a small proportion of the examined $\times 400$-magnified microscopic fields, 2 = single cells or aggregates observed in most of the examined $\times 400$-magnified microscopic fields, and $3=$ single cells or aggregates observed in roughly all of the examined $\times 400$ magnified microscopic fields.

\subsection{Antibodies}

Spleen cells were immunophenotyped using immunohistochemistry, as described by Silva et al. [21]. Briefly, serial spleen sections were placed on slides coated with poly-L-lysine (SigmaAldrich, United States) and dewaxed. Antigen retrieval was performed with citrate buffer at $\mathrm{pH} 6.0$ (for the anti-S100 and antiCD3 antibodies) or Tris-EDTA at pH 8.9 (for the anti-Ki67 antibody). For the remaining steps, the Novolink Max Polymer (Novocastra, United Kingdom) system was used following the protocol provided by the manufacturer. The slides were incubated for $1 \mathrm{~h}$ at $37^{\circ} \mathrm{C}$ with primary anti-CD3 (clone F7.2.38), anti-Ki67 (clone MIB-1), and a polyclonal anti-S100 antibody from Dako (Carpinteria, CA). In certain experiments, the positive controls consisted of human tissues known to be reactive with each marker. As a negative control, sections were incubated with buffer only or with an immunoglobulin of the same isotype as the test antibody but with different specificity. After staining, the sections were counterstained with hematoxylin, and the immunolabeling was evaluated microscopically [21].

\subsection{Expression and analysis of the results}

The data are presented in a figure and a table giving the relative and absolute values of the different parameters. Fisher's exact probability test was used to compare proportions between groups, and a Mann-Whitney test was used to compare scores between groups. Differences between groups were considered significant at $P<0.05$.

\section{Results}

\subsection{Diagnosis of infection and disease}

Clinical and laboratory data regarding infection and disease $(\mathrm{ZVL})$ are presented in Table 1 . All the animals in the infected group had positive serology. Leishmania was isolated from cultures of spleen aspirates from 5 animals. Amastigotes were visualized by histology of the spleen aspirates from 5 animals. Nine animals in the Leishmania-infected group had clinical signs of ZVL. Unfortunately, clinical information was not available for 4 animals in this group, all with serology or both serology and histology positive for Leishmania infection. The laboratory tests for detection of Leishmania infection were negative for the animals in the control group, and these animals did not present clinical signs of ZVL (Table 1).

\subsection{Spleen fragment features in cell-block preparations}

The $100-200 \mu \mathrm{L}$ of spleen aspirate collected from each dog provided an average of 18 slides suitable for histological analysis. The cell-block sections from the aspirates collected from uninfected dogs contained 33 [23-158] (median [1st quartile-3rd quartile]) spleen fragments, whereas those from infected animals contained 68 [23-254] spleen fragments (Table 2). The spleen fragment size varied from 22,945 [6040-60,261] $\mu \mathrm{m}$ to 310,646 $[163,497-1,926,099] \mu \mathrm{m}$ in the aspirates from the uninfected dogs and from $6890[1499-22,862] \mu \mathrm{m}$ to $311,641[17,276-749,743] \mu \mathrm{m}$ in the aspirates from the Leishmania-infected dogs. Smaller-sized fragments predominated in the aspirates from the infected dogs (Mann-Whitney test, $P<0.02$, Table 2). These spleen tissue fragments were dispersed among blood elements and isolated spleen cells (Fig. 1a).

\subsection{Qualitative and semi-quantitative analyses of cell-block preparations}

The red pulp and white pulp compartments were morphologically identified in the spleen aspirates (Fig. 1b-d). Red pulp and blood vessels were present in the cell-block preparations obtained from all sampled dogs (Table 2). The white pulp compartment was identified in all animals in the uninfected group and in 11 of the 13 animals in the infected group, which was a non-significant difference (Table 2). However, lymphoid follicles (Fig. 1c-d) were identified in 5 of 7 (71\%) uninfected specimens but only in 2 of 13 (15\%) specimens from Leishmania-infected dogs, which did represent a statistically significant difference (Fisher's test, $P<0.02$, Table 2). The usual cell constituents of the spleen (lymphocytes, macrophages, plasma cells, polymorphonuclear cells, and megakaryocytes) were easily identified in the tissue fragments and were also dispersed among the red blood cells. Granulomas were observed in 8 of 13 infected dogs and in none of the uninfected dogs (Fig. 1e). Leishmania amastigotes were observed in four samples, 
Table 2

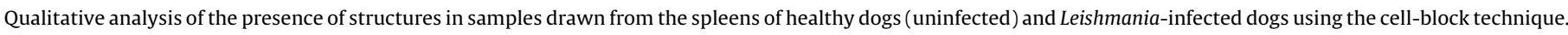

\begin{tabular}{|c|c|c|c|c|c|}
\hline \multirow[t]{2}{*}{ Parameter } & \multicolumn{4}{|c|}{ Evidence of Leishmania infection } & \multirow[t]{2}{*}{$P$} \\
\hline & NO & & YES & & \\
\hline \multicolumn{6}{|l|}{ Spleen fragments } \\
\hline$N$ & 33 & {$[23-158]^{\mathrm{a}}$} & 68 & {$[23-254]$} & 0.55 \\
\hline Smaller fragment size $\left(\mu \mathrm{m}^{2}\right)$ & 22,945 & {$[6,040-60,261]$} & 6,890 & {$[1,499-22,862]$} & $<0.02^{\mathrm{b}}$ \\
\hline Largest fragment size $\left(\mu \mathrm{m}^{2}\right)$ & 310,646 & {$[163,497-1,926,099]$} & 311,641 & {$[17,276-749,743]$} & 0.53 \\
\hline \multicolumn{6}{|l|}{ Red pulp ${ }^{\mathrm{d}}$} \\
\hline Frequency & $7 / 7$ & $(100 \%)$ & $13 / 13$ & $(100 \%)$ & 1.00 \\
\hline Macrophage score & 2.0 & {$[2.0-2.0]$} & 2.0 & {$[1.5-2.5]$} & 0.71 \\
\hline Lymphocyte score & 2.0 & {$[1.0-2.0]$} & 1.0 & {$[1.0-2.0]$} & 0.24 \\
\hline Plasma cell score & 1.0 & {$[1.0-1.0]$} & 3.0 & {$[1.5-3.0]$} & $<0.02^{\mathrm{c}}$ \\
\hline Neutrophil score & 2.0 & {$[1.0-2.0]$} & 2.0 & {$[1.0-2.0]$} & 0.83 \\
\hline Megakaryocytes & $6 / 7$ & $(86 \%)$ & $12 / 13$ & $(92 \%)$ & 1.00 \\
\hline Infected macrophages & $0 / 7$ & $(0 \%)$ & $5 / 13$ & $(38 \%)$ & 0.11 \\
\hline Granuloma & $0 / 7$ & $(0 \%)$ & $8 / 13$ & $(62 \%)$ & $<0.02$ \\
\hline Trabecle frequency & $5 / 7$ & $(71 \%)$ & $3 / 13$ & $(23 \%)$ & 0.06 \\
\hline Blood vessel frequency & $7 / 7$ & $(100 \%)$ & $13 / 13$ & $(100 \%)$ & 1.00 \\
\hline Sheathed capillaries & $4 / 7$ & $(57 \%)$ & $12 / 13$ & $(92 \%)$ & 0.10 \\
\hline \multicolumn{6}{|l|}{ White pulp } \\
\hline Frequency & $7 / 7$ & $(100 \%)$ & $11 / 13$ & $(85 \%)$ & 0.52 \\
\hline PALS & $2 / 7$ & $(27 \%)$ & $10 / 13$ & $(77 \%)$ & 0.06 \\
\hline Lymphoid follicle/Marginal zone & $7 / 7$ & $(100 \%)$ & $8 / 13$ & $(62 \%)$ & 0.11 \\
\hline Lymphoid follicle & $5 / 7$ & $(71 \%)$ & $2 / 13$ & $(15 \%)$ & 0.02 \\
\hline Marginal zone & $3 / 7$ & $(43 \%)$ & $1 / 13$ & $(8 \%)$ & 0.10 \\
\hline
\end{tabular}

all from dogs with positive spleen cultures (Fig. 1f). The plasma cell density score was higher in the spleens of infected dogs (3.0 [1.5-3.0]) than in those of uninfected dogs (1.0 [1.0-1.0]) (MannWhitney, $P<0.02$, Table 2, Fig. 1g). Furthermore, the combination of PAS and PAS-M staining allowed for differentiation between blood vessel- and muscle cell-associated basement membranes and the splenic reticular framework (Fig. 1h), occasionally disrupted by the presence of granulomas (Fig. 1i).

\subsection{Immunohistochemical staining for leukocyte subpopulation identification in cell blocks}

Immunohistochemical staining with monoclonal antibodies against CD3 (a T lymphocyte marker), S100 (a dendritic cell marker), and KI-67 (a cell proliferation marker) provided clear identification of cell subpopulations and allowed us to define their distributions in the spleen compartments (Fig. 1j-1). T lymphocytes predominated around arterioles (Fig. 1j). S100-positive cells formed a network around certain blood vessels (Fig. 1k). KI-67positive cells revealed proliferating cells distributed in the red and white pulp (Fig. 11)

\section{Discussion}

The cell-block technique described in the present report allows details of the spleen structure, such as blood vessels and spleen compartments, to be examined. This method also facilitates analysis of the distributions of leukocyte populations in these spleen compartments, without the confounding interference of circulating blood cells, which aids in the identification of pathological structures such as granulomas. Such spleen structure details may be further characterized using histochemical staining of the connective matrix scaffolding, such as with PAS and PAS-M, and immunohistochemistry for cell phenotyping. The presence of white pulp, though less frequent in the Leishmania-infected dogs, did not differ significantly between the infected and the uninfected groups. It is noteworthy that the presence of lymphoid follicles was less frequent in the Leishmania-infected group, which may correspond to an increased spleen size due to a relative increase in red pulp as well as lymphoid follicle atrophy and disruption, both of which are observed in dogs with ZVL $[12,21,22]$. Furthermore, disrupted lymphoid follicles may be overlooked in the cell-block analysis, as may also occur when analyzing conventional histological spleen sections. The increased presence of small spleen fragments in the aspirates collected from the infected group may correspond to a change in the consistency of the spleen, which may become friable in dogs with ZVL. In fact, inflammation and an increase in spleen size may also disrupt the relationship between the cell and the extracellular matrix, altering spleen consistency. For instance, the presence of trabeculae was less frequent in Leishmania-infected dogs' aspirates than in uninfected dogs' aspirates, and the framework of the PAS-M-positive reticulum was altered by the presence of granulomas.

A striking finding of this study was the presence of granulomas and clumps of infected macrophages in the red pulp of certain infected dogs. These observations would not have been possible using conventional cytology. This finding is relevant to the nature of the dog immune response to Leishmania infection [12,22]. In fact, a variety of disturbances to the host immune response to Leishmania and other intracellular pathogens may cause different patterns of macrophage aggregation and granuloma characteristics [10,23-25].

Using this cell-block technique, we were able to access the cellular constitution of spleen compartments using conventional histology or immunohistochemistry. We confirmed an increase in plasma cell density in the spleens of Leishmania-infected dogs. In fact, splenic white pulp disruption and plasmacytosis are present in dogs with severe $\mathbf{Z V L}$ [12-14]. These changes are associated with the abnormal expression of cytokines that are responsible for lymphoid follicle organization and plasma cell homing and survival, such as CXCL-13, BAFF, and APRIL [21] (Silva, unpublished data).

Finally, the cell-block technique presented here may be used to build a panel of tests to be employed in the follow-up care and study of dogs with ZVL and other diseases affecting the spleen in both veterinary clinics and research settings. Using the cell-block technique together with immunohistochemistry, laser microdis- 

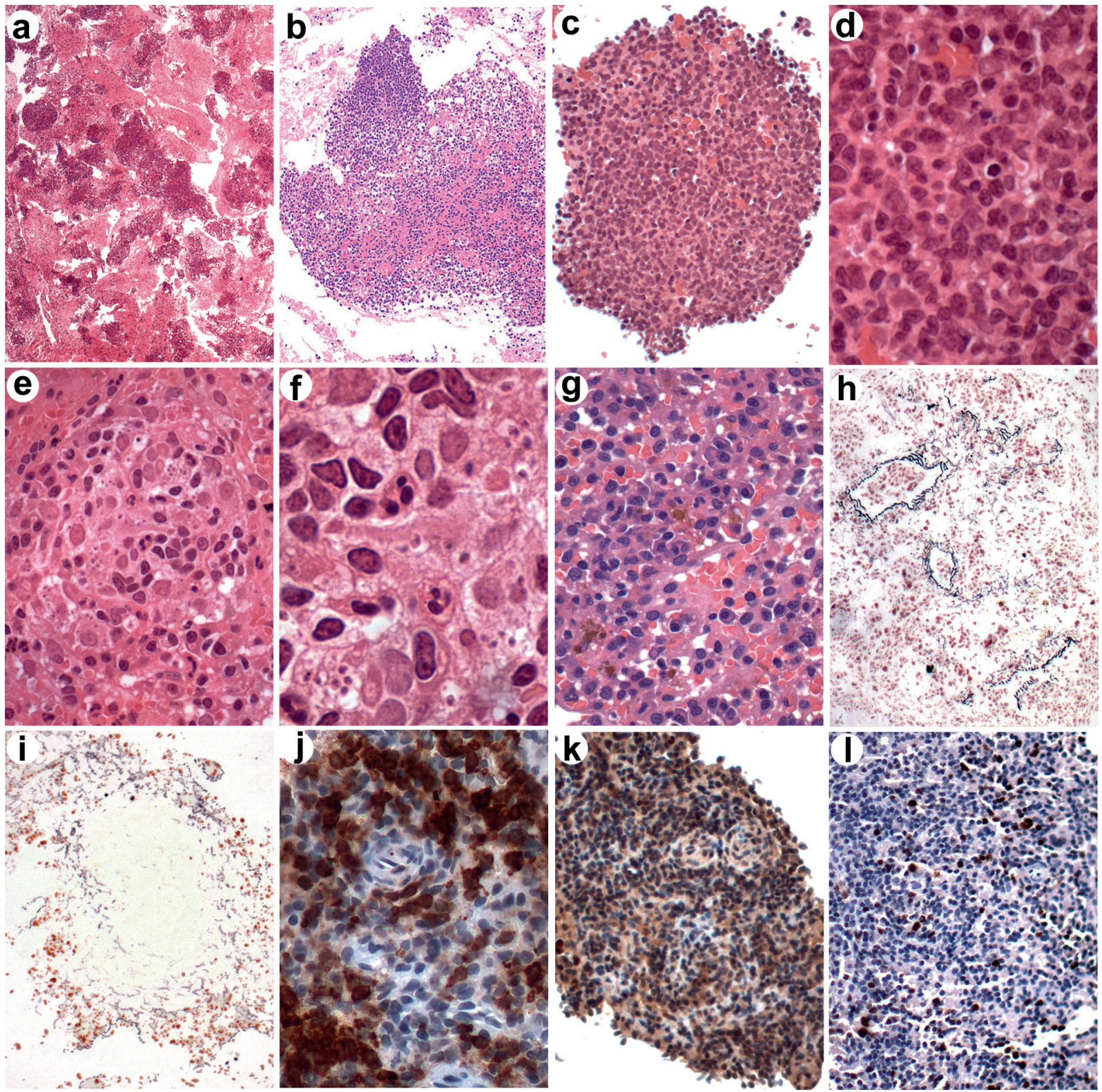

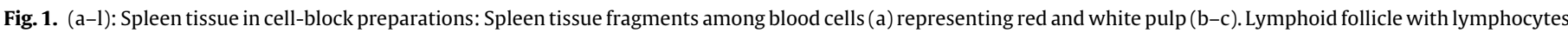

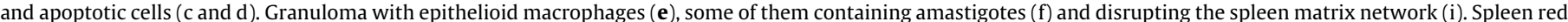

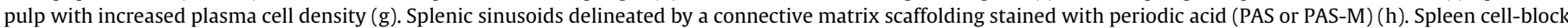

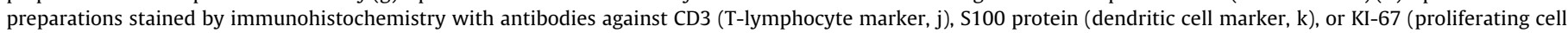
marker, 1). (For interpretation of the references to colour in this figure legend, the reader is referred to the web version of this article.)

section, and genetic analysis may provide a better understanding of the pathogenesis of $\mathbf{Z V L}$ and other diseases in which the spleen plays a central role.

\section{Conclusions}

The cell-block technique described herein allows details of spleen histology to be studied;

The method also allows comparative studies between healthy dogs and those with diseases affecting the spleen, without needing to euthanize the animals or to subject them to splenectomies; and

Given its minimally invasive characteristic, this technique can be used in follow-up studies of diseases affecting the spleen in both research and veterinary practice.

\section{Acknowledgments}

We thank DVM Jamille Nonato Amaral for veterinary support. This work was supported by Fundação de Amparo à Pesquisa do Estado da Bahia (Fapesb. http://www.fapesb.ba.gov.br) grant no. PET0053/2013, AUXPE-CAPES-FAPESB 2072/2013/PROCESSO \#23038.006718/2013-19 and Fundação Oswaldo Cruz (Fiocruz. http://portal.fiocruz.br/pt-br)-CNPq PROEP grant 400905/2013-2. WLCS received a scholarship from CNPq. The funders had no role in the study design, data collection or analysis, decision to publish, or preparation of the manuscript.

\section{References}

[1] M.F. Cesta, Normal structure, function, and histology of the spleen, Toxicol. Pathol. 34 (5) (2006) 455-465. 
[2] A.M. van der Laan, E.N. Ter Horst, R. Delewi, M.P. Begieneman, P.A. Krijnen, A. Hirsch, M. Lavaei, M. Nahrendorf, A.J. Horrevoets, H.W. Niessen, J.J. Piek, Monocyte subset accumulation in the human heart following acute myocardial infarction and the role of the spleen as monocyte reservoir, Eur. Heart J. 35 (6) (2014) 376-385.

[3] B. Steiniger, P. Barth, Microanatomy and function of the spleen, Adv. Anat. Embryol. Cell Biol. 151 (III-IX) (2000) 1-101.

[4] V.E. Valli, P.H. Kass, M. San Myint, F. Scott, Canine lymphomas: association of classification type, disease stage, tumor subtype, mitotic rate, and treatment with survival, Vet. Pathol. 50 (5) (2013) 738-748.

[5] K. Hansen, D.B. Singer, Asplenic-hyposplenic overwhelming sepsis: postsplenectomy sepsis revisited, Pediatr. Dev. Pathol. 4 (2) (2001) 105-121.

[6] A.T. Camacho, E. Pallas, J.J. Gestal, F.J. Guitian, A.S. Olmeda, Babesia canis infection in a splenectomized dog, Bull. Soc. Pathol. Exot. 95 (1) (2002) 17-19.

[7] J. Carrion, A. Nieto, S. Iborra, V. Iniesta, M. Soto, C. Folgueira, D.R. Abanades, J.M. Requena, C. Alonso, Immunohistological features of visceral leishmaniasis in BALB/c mice, Parasite Immunol. 28 (5) (2006) 173-183.

[8] R. Badaro, E. Falcoff, F.S. Badaro, E.M. Carvalho, D. Pedral-Sampaio, A. Barral, J.S. Carvalho, M. Barral-Netto, M. Brandely, L. Silva, et al., Treatment of visceral leishmaniasis with pentavalent antimony and interferon gamma, New Engl. J. Med. 322 (1) (1990) 16-21.

[9] W.L. Dos-Santos, C. Pagliari, L.G. Santos, V.A. Almeida, T.L. e Silva, J. Coutinho Jde Jr., T. Souza, M.I. Duarte, L.A. de Freitas, C.H. Costa, A case of conventiona treatment failure in visceral leishmaniasis: leukocyte distribution and cytokine expression in splenic compartments, BMC Infect. Dis. 14 (2014) 491.

[10] M.E. Wilson, M. Sandor, A.M. Blum, B.M. Young, A. Metwali, D. Elliott, R.G. Lynch, J.V. Weinstock, Local suppression of IFN-gamma in hepatic granulomas correlates with tissue-specific replication of Leishmania chagasi, J. Immunol. 156 (6) (1996) 2231-2239.

[11] R.A. Dutra, L.F. Dutra, O. Reis Mde, R.C. Lambert, Splenectomy in a patient with treatment-resistant visceral leishmaniasis: a case report, Rev. Soc. Bras. Med. Trop. 45 (1) (2012) 130-131.

[12] C.C. Santana, J. Vassallo, L.A. de Freitas, G.G. Oliveira, L.C. Pontes-de-Carvalho, W.L. dos-Santos, Inflammation and structural changes of splenic lymphoid tissue in visceral leishmaniasis: a study on naturally infected dogs, Parasite Immunol. 30 (10) (2008) 515-524.

[13] I.S. Lima, J.S. Silva, V.A. Almeida, F.G. Junior, P.A. Souza, D.F. Larangeira, J.P. Moura-Neto, D.B. Fraga, L.A. de Freitas, W.L. dos-Santos, Severe clinical presentation of visceral leishmaniasis in naturally infected dogs with disruption of the splenic white pulp, PLoS One 9 (2) (2014) e87742.

[14] V.M. de Lima, K.R. Fattori, F. de Souza, F.R. Eugenio, P.S. dos Santos, D.B. Rozza, G.F. Machado, Apoptosis in T lymphocytes from spleen tissue and peripheral blood of L. (L.) chagasi naturally infected dogs, Vet. Parasitol. 184 (2-4) (2012) 147-153.
[15] A.S. Cavalcanti, M. Ribeiro-Alves, O. Pereira Lde, G.L. Mestre, A.B. Ferreira, F.N Morgado, M.C. Boite, E. Cupolillo, M.O. Moraes, R. Porrozzi, Parasite load induces progressive spleen architecture breakage and impairs cytokine mRNA expression in Leishmania infantum-naturally infected dogs, PLoS One 10 (4) (2015) e0123009.

[16] B. Veress, A. Omer, A.A. Satir, A.M. El Hassan, Morphology of the spleen and lymph nodes in fatal visceral leishmaniasis, Immunology 33 (5) (1977) 605-610.

[17] S.M. Barrouin-Melo, D.F. Larangeira, F.A. de Andrade Filho, J. Trigo, F.S. Juliao, C.R. Franke, P.H. Palis Aguiar, W.L. Conrado dos-Santos, L. Pontes-de-Carvalho, Can spleen aspirations be safely used for the parasitological diagnosis of canine visceral leishmaniosis? A study on assymptomatic and polysymptomatic animals, Vet. J. 171 (2) (2006) 331-339.

[18] S.M. Barrouin-Melo, D.F. Larangeira, J. Trigo, P.H. Aguiar, W.L. dos-Santos, L. Pontes-de-Carvalho, Comparison between splenic and lymph node aspirations as sampling methods for the parasitological detection of Leishmania chagasi infection in dogs, Mem. Inst. Oswaldo Cruz 99 (2) (2004) 195-197.

[19] S.M. Barrouin-Melo, D.F. Larangeira, S.O. Santos, A.D. Chagas-Junior, M. Paixao, P.H. Aguiar, W.L. dos-Santos, L. Pontes-de-Carvalho, A standardized cytological and immunochemical method for the analysis of fine-needle spleen aspirates: assessment of leukocyte population changes in canine visceral leishmaniosis, Vet. Immunol. Immunopathol. 111 (3-4) (2006) 251-261.

[20] W.L. Dos-Santos, E.E. Jesus, M. Paranhos-Silva, A.M. Pereira, J.C. Santos, C.O. Baleeiro, E.G. Nascimento, E.D. Moreira, G.G. Oliveira, L.C. Pontes-de-Carvalho, Associations among immunological, parasitological and clinical parameters in canine visceral leishmaniasis: emaciation, spleen parasitism, specific antibodies and leishmanin skin test reaction, Vet. Immunol. Immunopathol. $123(3-4)$ (2008) 251-259.

[21] J.S. Silva, A.C. Andrade, C.C. Santana, L.Q. Santos, C.I. Oliveira, P.S. Veras, J. Vassallo, W.L. dos-Santos, Low CXCL13 expression, splenic lymphoid tissue atrophy and germinal center disruption in severe canine visceral leishmaniasis, PLoS One 7 (1) (2012) e29103.

[22] G.G. Oliveira, F. Santoro, M. Sadigursky, The subclinical form of experimental visceral leishmaniasis in dogs, Mem. Inst. Oswaldo Cruz 88 (2) (1993) 243-248.

[23] G. Fenhalls, L. Stevens, J. Bezuidenhout, G.E. Amphlett, K. Duncan, P. Bardin, P.T. Lukey, Distribution of IFN-gamma, IL-4 and TNF-alpha protein and CD8 T cells producing IL-12p40 mRNA in human lung tuberculous granulomas, Immunology 105 (3) (2002) 325-335.

[24] H.W. Murray, Tissue granuloma structure-function in experimental visceral leishmaniasis, Int. J. Exp. Pathol. 82 (5) (2001) 249-267.

[25] W.L. dos-Santos, J. David, R. Badaro, L.A. de-Freitas, Association between skin parasitism and a granulomatous inflammatory pattern in canine visceral leishmaniosis, Parasitol. Res. 92 (2) (2004) 89-94. 